\title{
Measurement of the Translational Energy of Ions with a Time-of-Flight Mass Spectrometer
}

\author{
J. L. Frankin, Peter M. Hierd, ${ }^{*}$ and David A. Whan $\dagger$ \\ Department of Chemistry, Rice University, Houston, Texas
}

(Received 5 May 1967)

\begin{abstract}
A mathematical analysis of peak shapes in a Bendix time-of-flight mass spectrometer shows that ions formed with excess translational energy exhibit considerable peak broadening. A method is developed by which this translational energy can be readily determined from the peak width. Experimental results are presented which confirm the validity of the theoretical calculations.
\end{abstract}

\section{INTRODUCTION}

Fragment ions formed with excess translational energy have long been observed in the mass spectra of many molecules. Accurate measurements of these excess energies are often necessary (as, for example, in conjunction with appearance potential measurements) and, consequently, have been undertaken by a number of investigators in the field.

The detection and measurement of initial translational energy usually involves one of two basic methods, the use of retarding potentials, ${ }^{1-8}$ or an analysis of the focusing properties of ions formed with translational energy..$^{-16}$ These techniques, however, have all been developed either for conventional magnetic mass spectrometers or for specially designed instruments. Consequently, the present study was undertaken to develop a method by which these measurements could be made with a time-of-flight (TOF) mass spectrometer.

Wiley and McLaren ${ }^{17}$ have given a detailed analysis of the focusing action of a TOF mass spectrometer, and it is this treatment which has been extended to permit the measurement of the initial translational energies of ions by an analysis of their peak shapes. The feasibility of such an approach had previously

\footnotetext{
* Present address: Department of Chemistry, Yale University, New Haven, Conn.

$\dagger$ Present Address: Department of Chemistry, University of Edinburgh, Edinburgh, Scotland.

1 J. T. Tate and W. W. Lozier, Phys. Rev. 39, 254 (1932)

2 W. Bleakney, Phys. Rev. 35, 1180 (1930).

3 E. E. Hanson, Phys. Rev. 51, 86 (1937).

4 R. K. Curran and R. E. Fox, J. Chem. Phys. 34, 1590 (1961)

5 R. E. Fox and J. A. Hipple, Rev. Sci. Instr. 19, 462 (1948).

6 H. D. Hagstrum, Rev. Mod. Phys. 23, 185 (1951).

7 R. J. Kandel, J. Chem. Phys. 23, 84 (1955).

${ }^{8}$ H. E. Stanton and S Wexler, J. Chem. Phys. 44, 2959 (1966).

- C. E. Berry, Phys. Rev. 78, 597 (1950).

$10 \mathrm{G}$. Careri and G. Nencini, J. Chem. Phys. 18, 897 (1950).

1 N. D. Coggeshall. J. Chem. Phys. 12, 19 (1944).

12 N D. Coggeshall, J. Chem. Phys. 36, 1640 (1962)

13 H. D. Hagstrum and J. T. Tate, Phys. Rev 59, 354 (1941).

${ }_{14}$ A. Hustrulid, P. Kusch, and J. T. Tate, Phys. Rev. 54, 1037 (1938).

${ }^{16} \mathrm{C}$. A. McDowell and J. W. Warren, Discussions Faraday Soc. 10, 53 (1951).

${ }_{16}$ R. M. Reese and J. A. Hipple, Phys. Rev. 75, 1332 (1949).

17 W. C. Wiley and I. H. McLaren, Rev. Sci. Instr. 26, 1150 (1955).
}

been discussed in a qualitative manner by Ferguson et $a l^{18}$

\section{EXPERIMENTAL}

Measurements were made on a Bendix time-of-flight mass spectrometer, Model 12, using an S14-107 ion source and a flight tube of $180 \mathrm{~cm}$. The scanning device on one analog unit was modified by the insertion of a 1-M $\Omega$ resistor in the scan rate control circuit. This modification provided an extremely slow scan rate which, in conjunction with a Honeywell chart recorder running at 8 in. per min, enabled $10^{-8} \mathrm{sec}$ of real time to be displayed by 0.328 in. of chart paper. To ensure optimum resolution the ion lens was not used when recording peak shapes.

\section{FLIGHT TIMES}

Figure 1 shows the major elements of a TOF mass spectrometer. There are three principal regions: the ionization region, bounded by the backing plate and the ion focus grid; the acceleration region, bounded by the ion focus grid and the ion energy grid; and the drift tube, a field-free region extending from the energy grid to the collector cathode.

The pulsed electron beam is collimated by a system of slits and has a small but finite thickness, $\Delta s$. The center of the electron beam is at a distance $s_{0}$ from the ion focus grid. Immediately after the electron beam is turned off, the ion focus grid receives a pulse of from 0 to $-250 \mathrm{~V}$. The resulting electric field $E_{s}$ draws the positive ions out of the ionization region into the acceleration region. The ion energy grid is maintained at $-3000 \mathrm{~V}$, resulting in an electric field $E_{d}$ in the acceleration region.

An ion of charge $q$, formed in the source at distance $s$ from the ion focus grid with a component of initial translational energy $U_{x}$ parallel to the axis of the flight tube, will have a total flight time given by

$$
T\left(U_{x}, s\right)=T_{s}\left(U_{x}, s\right)+T_{d}\left(U_{x}, s\right)+T_{D}\left(U_{x}, s\right),
$$

${ }^{18}$ R. E. Ferguson, K. E. McCulloh, and H. M. Rosenstock, 148 
where

$$
\begin{gathered}
T_{s}\left(U_{x}, s\right)=\left[(2 m)^{1 / 2} / q E_{s}\right]\left[\left(q s E_{s}+U_{x}\right)^{1 / 2} \pm\left(U_{x}\right)^{1 / 2}\right] \\
T_{d}\left(U_{x}, s\right)=\left[(2 m)^{1 / 2} / q E_{d}\right]\left[\left(q d E_{d}+q s E_{s}+U_{x}\right)^{1 / 2}\right. \\
\left.-\left(q s E_{s}+U_{x}\right)^{1 / 2}\right], \\
T_{D}\left(U_{x}, s\right)=(2 m)^{1 / 2}\left[D / 2\left(q d E_{d}+q s E_{s}+U_{x}\right)^{1 / 2}\right] .
\end{gathered}
$$

The plus and minus sign in (2a) correspond to initial velocities directed away from and toward the collector, respectively.

\section{RESOLUTION}

If all of the ions were formed at rest $\left(U_{x}=0\right)$, and if they were all in a plane parallel to the ion focus grid $\left(s=s_{0}\right)$, then the flight times would depend upon the mass to charge $(m / e)$ ratio only. Every ion of a given $m / e$ would arrive at the collector at exactly the same instant, resulting in a mass spectrum consisting entirely of sharp lines or delta functions at the appropriate mass numbers. Any variation in initial energy and/or initial position would result in a slight variation in the flight time and a consequent broadening of the ion peak. The effects of peak broadening due to variations in initial position and in initial energy are referred to as the problems of space resolution and energy resolution, respectively.

\section{A. Space Resolution}

Instead of being formed in a plane parallel to the ion focus grid, the ions are actually formed throughout the entire thickness of the electron beam $\Delta s$. If $\Delta T_{\Delta s}$, the change in flight time caused by a small change $\Delta s$ in the position variable $s$, is defined by the expression

$$
\Delta T_{\Delta_{\mathrm{s}}}=T\left(0, s_{0}+\Delta s\right)-T\left(0, s_{0}\right),
$$

then expansion of $T\left(0, s_{0}+s\right)$ in a MacLaurin series about $s_{0}(17)$ yields the expression

$$
\Delta T_{\Delta s}=\sum_{n=1}^{\infty} \frac{1}{n !}\left[\frac{d^{n} T(0, s)}{d s^{n}}\right]_{s 0}(\Delta s)^{n} .
$$

The effect of these variations in the initial positions of the ions is counteracted by the fact that ions farther away from the ion focus grid fall through a larger potential when accelerated by the focus pulse than do those ions formed nearer to the ion focus grid. Because of their greater energy, the former eventually overtake and pass the latter at some distance down the flight tube. By a careful adjustment of the focus pulse, this "crossover point" can be made to occur just at the collector cathode. Calculation of the first three terms of the rapidly converging series in Eq. (4) shows that $\Delta T_{\Delta_{s}}$ goes through zero at a focus pulse of $-120 \mathrm{~V}$ for the ion source used. Focus pulse values smaller than this do not permit the crossover to occur before the ions reach the collector, while larger values will cause

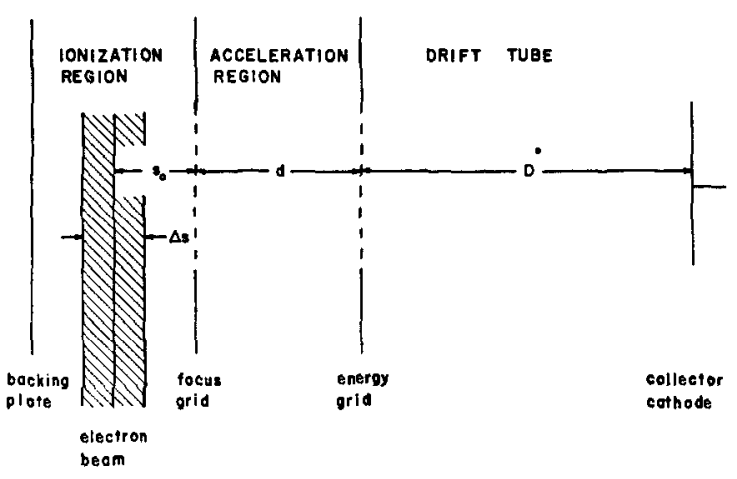

FIG. 1. Schematic representation of the time-of-flight mass spectrometer.

the cross-over to occur prematurely in the drift tube. A value of $-120 \mathrm{~V}$, however, eliminates the effect of variations in initial position, so that the broadening of the ion packet is caused by variations in initial translational energy only.

\section{B. Energy Resolution}

Consider the case of two identical ions formed at the same initial position $s$ with equal but oppositely directed velocity components in the $x$ direction (parallel to the axis of the flight tube).

$$
\begin{aligned}
&-v_{x}-(1) \\
&(2) \rightarrow+v_{x} .
\end{aligned}
$$

Upon its formation ion (1) moves away from the collector, decelerating due to the electric field $E_{8}$. At time $\Delta T=m v_{x} / q E_{s}$, ion (1) comes to rest, reverses direction, and is accelerated towards the collector, returning to its original position with a velocity in the $x$ direction equal in magnitude to its original $x$ velocity, but this time directed toward the collector. Subsequently, its motion is identical to that of ion (2), which it continues to lag behind in time by twice the deceleration time $m v_{x} / q E_{s}$. An ion formed with zero initial velocity in the $x$ direction will have a flight time midway between those of ions (1) and (2), so that

$$
T\left(U_{x}, s\right)=T(0, s) \pm \Delta T_{U}
$$

where

$$
\Delta T_{U}=m v_{x} / q E_{8}=\left[(2 m)^{1 / 2} / q E_{\mathrm{s}}\right]\left(U_{x}\right)^{1 / 2},
$$

and the plus and minus signs refer to initial velocities directed away from and toward the collector, respectively.

The flight time is independent of velocity components perpendicular to the $x$ axis. Moreover, because the ion beam is not collimated by a system of slits and because the area of the collector cathode is large compared to the height of the electron beam, there is no discrimination against high-energy ions. That is, the collection efficiency and, hence, the relative intensity of 


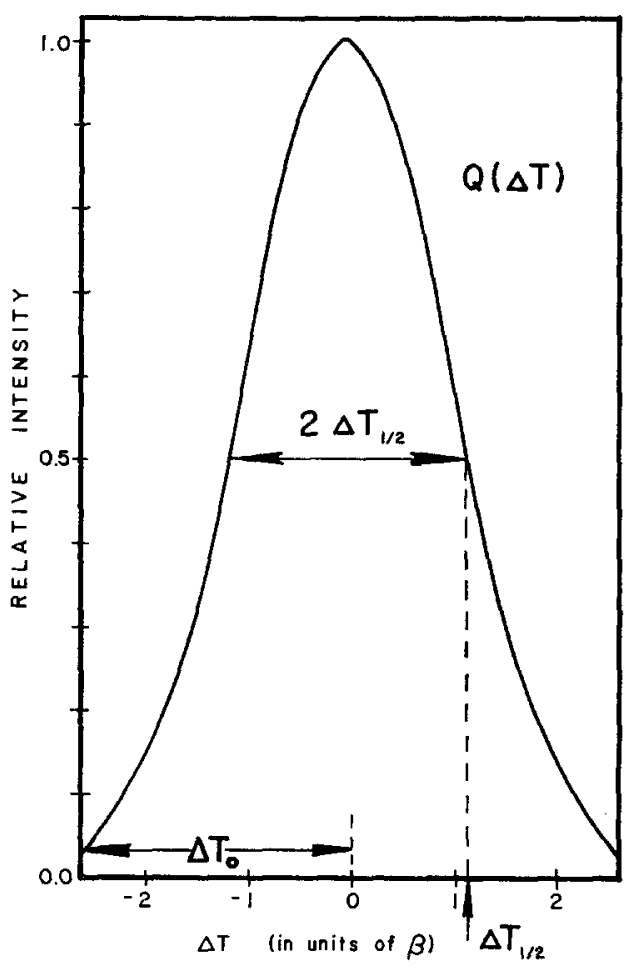

FIG. 2. Calculated peak shape for ions with thermal energy.

the ion peak is independent of any velocity components in the $y$ or the $z$ direction.

\section{PEAK SHAPES}

If the focus condition is obeyed $\left(\Delta T_{\Delta A}=0\right)$ and the operating conditions are kept constant, then the total flight time will depend upon the initial velocity in the $x$ direction for an ion of given $m / e$. If the probability distribution for the component of initial velocity along the direction of the flight tube is known, the probability distribution for $\Delta T_{U}$ can be obtained. This will permit the calculation of the relative intensity of the ion signal as a function of $\Delta T_{U}$ and consequently a calculation of the shape of the ion peak. If $P\left(v_{x}\right)$ is a known distribution function for the variable $v_{x}$, and $v_{x}=f\left(\Delta T_{U}\right)$, then the corresponding distribution function in the variable $\Delta T_{U}$ is

$$
Q\left(\Delta T_{U}\right)=P\left[f\left(\Delta T_{U}\right)\right] f^{\prime}\left(\Delta T_{U}\right) .
$$

From Eq. (6)

$$
\begin{aligned}
f^{\prime}\left(\Delta T_{U}\right) & =\left[d / d\left(\Delta T_{U}\right)\right]\left(q E_{s} / m\right)\left(\Delta T_{U}\right) \\
& =q E_{s} / m .
\end{aligned}
$$

The function $Q\left(\Delta T_{U}\right)$ represents the probability that an ion of given $m / e$ will have a total flight time differing by a small amount $\Delta T_{U}$ from the flight time of an identical ion formed with zero initial translational energy $T\left(0, s_{0}\right)$. It is assumed that the focus condition is obeyed so that all ions act as if they are formed at $s_{0}$.
The function $Q\left(\Delta T_{U}\right)$, if known, permits the calculation of the initial translational energy of the ion from the peak shape.

\section{A. Thermal Ions}

For ions with a thermal or quasithermal energy distribution, the component of velocity in a given direction has a Gaussian distribution:

$P\left(v_{x}\right)=\left(A \pi^{1 / 2}\right)^{-1} \exp \left(-v_{x}^{2} / A^{2}\right), \quad A=(2 k T / m)^{1 / 2}$,

where $k$ is the Boltzmann constant, and $T$ is the absolute temperature. The probability distribution for $\Delta T_{U}$ is obtained by making the appropriate change of variables:

$$
\begin{aligned}
Q\left(\Delta T_{U}\right) & =\left(B \pi^{1 / 2}\right)^{-1} \exp \left[-\left(\Delta T_{U}\right)^{2} / B^{2}\right] \\
B & =\left(m / q E_{s}\right) A
\end{aligned}
$$

For ions of a given mass and a thermal or nearly thermal distribution of energies, the intensity of the ion signal as a function of flight time will have a Gaussian distribution about the average value, $T\left(0, s_{0}\right)$. (See Fig. 2.)

From the known distribution it is possible to calculate the time at which the intensity has fallen to one-half of its maximum value and to express this time $\left(\Delta T_{1 / 2}\right)$ in terms of the constant $B$, which in turn is a function of the parameters $q, m, E_{s}$, and $k$, and of the temperature of the gas $T$, from which one can determine the average value of the initial translational energy $\bar{U}$.

The time at which the ion peak has fallen to one-half of its maximum value is obtained from the expression

$$
\exp \left[-\left(\Delta T_{1 / 2}\right)^{2} / B^{2}\right]=\frac{1}{2}
$$

which yields

$$
\left[\Delta T_{1 / 2} / B\right]^{2}=\ln 2 .
$$

Equivalently, the relationship can be expressed in terms of the full width at half-height $W_{1 / 2}$ a quantity which is more conveniently measured experimentally. Since the ion peak is symmetric,

$$
W_{1 / 2}=2 \Delta T_{1 / 2}=2(\ln 2)^{1 / 2} B \text {. }
$$

From Eq. (9) and Eq. (10)

$$
W_{1 / 2}=1.664(2 k T m)^{1 / 2} / q E_{s} .
$$

With $\bar{U}=(3 / 2) k T$, the average value of the initial translational energy for a thermal distribution is given by

$$
\bar{U}=\left(N_{0} / 3.69\right)\left(q E_{s}\right)^{2}\left[\left(W_{1 / 2}\right)^{2} / M\right],
$$

where $N_{0}$ is Avogadro's number and $M$ is the molecular weight of the ion in grams per mole.

\section{B. Excess-Energy Ions}

The distribution of initial velocities for ions formed by electron-impact induced ionization and dissociation depends upon the potential energy curves for the states involved. The most probable value for the initial energy 
could be anywhere from zero to several electron volts, depending upon the relative positions of the potentialenergy curves. Moreover, this excess energy could be distributed over a narrow or a wide range of values, depending upon the slope of the upper curve as it passes through the Franck-Condon region.

Since the present analysis of peak shapes determines the probability distribution for a given component of initial velocity rather than the distribution for the total initial speed (i.e., energy), it is quite difficult to interpret the resulting peak shapes without prior knowledge of the potential-energy curves. For example, an ion appearing at time $T\left(0, s_{0}\right)$ may either have been formed with zero initial energy or have been formed with considerable excess energy directed perpendicularly to the subsequent flight path. Because the ions are not collimated by slits, there is no discrimination against ions formed with nonzero components of velocity perpendicular to the flight tube.

When the dissociation process yields ions with a high initial translational energy that is distributed over a relatively narrow range, the resulting ion peak is nearly rectangular. This can be shown if one considers the case in which a number of ions are formed at the same point with equal but randomly directed velocities $v_{0}$. The velocity vectors will form a uniform sphere of radius $v_{0}$. The probability that the component of velocity in a given direction (e.g., $v_{x}$ along the flight tube) has a given velocity in the range between $v_{x}$ and $v_{x}+d v_{x}$ is simply given by the area of the sphere's surface lying between a plane perpendicular to and intersecting the $x$ axis at distance $v_{x}$ from the origin, and a second such perpendicular plane at distance $v_{x}+d v_{x}$ from the origin. That is, an ion whose velocity vector ends on the surface of this zone will have a component of velocity along the $x$ axis in the desired range, $v_{x}$ to $v_{x}+d v_{x}$. The probability, $P\left(v_{x}\right) d v_{x}$, that the velocity component is in this range is given by the ratio of the area of this zone to the total area of the sphere; that is

$$
\begin{aligned}
P\left(v_{x}\right) d v_{x} & =2 \pi v_{0} d v_{x} / 4 \pi v_{0}^{2} \quad-v_{0} \leq v_{x} \leq v_{0} \\
& =\left(2 v_{0}\right)^{-1} d v_{x} .
\end{aligned}
$$

Combining Eqs. (7), (8), and (16) results in the equation

$$
Q(\Delta T)=\left[2\left(\Delta T_{0}\right)\right]^{-1} \quad-\Delta T_{0} \leq \Delta T \leq \Delta T_{0},
$$

where

$$
\Delta T_{0}=m v_{0} / e E_{s} .
$$

This type of distribution function results in ion peaks rectangular in shape. Ion peaks very similar to this shape have been observed experimentally (see for example the lower part of the experimental trace in Fig. 5), indicating that the fragment ions under observation were formed by a process which gives them all the same or very nearly the same amount of initial

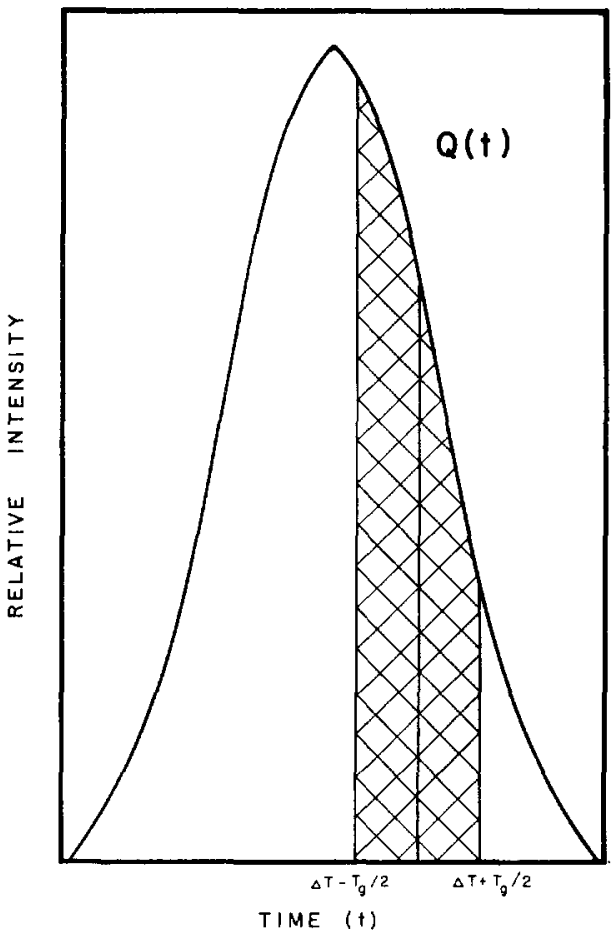

FiG. 3. Schematic illustration of the effect of the finite gate width.

energy $U_{0}$. Since $U_{0}$ equals $\frac{1}{2} m v_{0}^{2}$, and the relationship between the experimentally observed peak width and the velocity is given by Eq. (18), one can determine the translational energy with which the ions are formed by a measurement of the peak width. For the sake of convenience, one defines $W_{0}$, the full width of the ion peak at its base, by the equation

$$
\begin{aligned}
W_{0}=2 \Delta T_{0} & =2 m v_{0} / q E_{s} \\
& =\left[2(2 m)^{1 / 2} / q E_{s}\right]\left(U_{\max }\right)^{1 / 2},
\end{aligned}
$$

which yields the relationship

$$
U_{\max }=\frac{1}{8} N_{0}\left(e E_{s}\right)^{2}\left(W_{0}^{2} / M\right)=\frac{1}{2} m v_{0}^{2} .
$$

The procedure for all ions whose energy distributions are clearly not thermal or quasithermal has been to measure the width at the base of the peak $\left(W_{0}\right)$. This, with Eq. (20) gives the maximum energy for a detectable number of ions having a large amount of excess energy distributed over a relatively small range of energies.

\section{Effect of Gate Width}

An additional factor that affects the peak shape is the manner in which the instrument itself detects and records the ion signal. For this discussion a clear distinction must be drawn between the actual ion beam or packet and its representation (the ion signal) as produced by the instrument.

When an ion packet strikes the collector cathode, electrons are knocked from its surface and are directed 


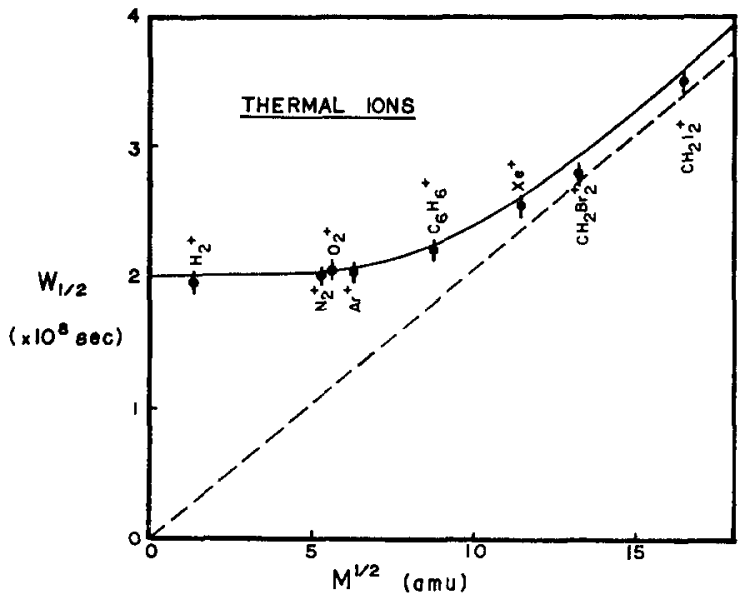

Fig. 4. Calculated and experimental peak widths for ions of known (i.e., thermal) energy. The dotted line represents the full width at half-maximum calculated without taking into account the widening effect of the finite gate pulse [Eq. (14)]. The solid line represents the FWHM obtained by the construction of a number of hypothetical peaks from $\mathrm{Eq}$. (19). The points represent experimentally determined peak widths for a number of ions of thermal energy.

onto the multiplier. The current produced in the multiplier by an individual ion packet is measured by deflecting the electron pulse for that mass from the electron-pulse train (normally going to this oscilloscope anode) to one of the gated anodes of the multiplier.

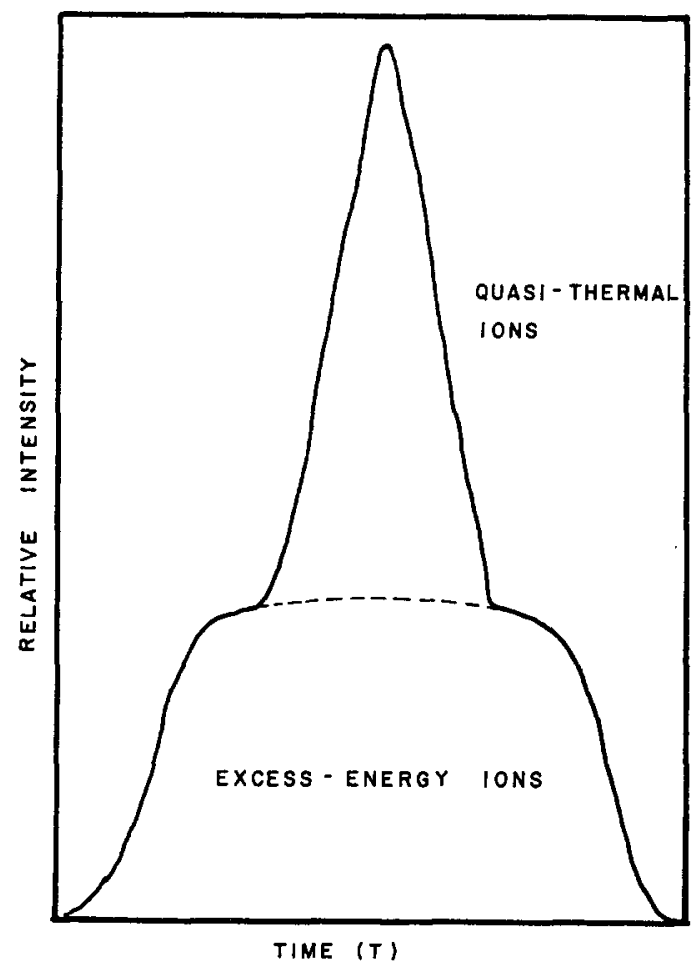

Fig. 5. Chart recording of the methyl ion obtained from the dissociation of ethyl bromide. Note that the upper quasithermal portion of the trace must be corrected for the effect of gate width before it can be compared with the lower, high-energy portion.
TABLE I. Translational energies of $\mathrm{CH}_{2}+$ fragment ions from $\mathrm{C}_{2} \mathrm{H}_{6} \mathrm{Br}$.

\begin{tabular}{lcc}
\hline \hline & This work & $\begin{array}{c}\text { Olmsted, Street, } \\
\text { and Newton* }\end{array}$ \\
\hline Quasithermal ion & $0.15 \mathrm{eV}$ & $0.21 \mathrm{eV}$ \\
Excess-energy ion & $3.25 \mathrm{eV}$ & $3.31 \mathrm{eV}$ \\
\hline
\end{tabular}

Reference 22.

This is accomplished by precisely timed pulses which gate the desired mass peak to the appropriate anode. The effect of this gate pulse is that instead of measuring the intensity of the ion beam at some particular time $Q\left(\Delta T_{U}\right)$, the instrument actually records the sum of the signals for all ions that have arrived at the collector over the finite period of time that the anode is gated. (See Fig. 3.) Consequently, the instrument records an ion signal whose relative intensity as a function of time is given by the integral

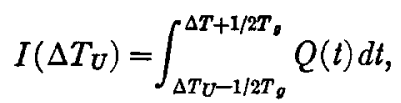

where $Q(t)$ is the distribution function defined in $\mathbf{E q}$. (7), which gives the intensity of the ion beam at some particular time. The duration of the gate pulse $T_{g}$ was estimated to be 20 nsec.

For thermal ions, which have the Gaussian distribution function shown in Eq. (10), the integral can be broken down into two simpler integrals:

$$
\begin{array}{r}
I\left(\Delta T_{U}\right)=\int_{0}^{x_{2}}\left[(2 \pi)^{1 / 2}\right]^{-1} \exp \left(-\frac{1}{2} x^{2}\right) d x \\
\quad-\int_{0}^{x_{1}}\left[(2 \pi)^{1 / 2}\right]^{-1} \exp \left(-\frac{1}{2} x^{2}\right) d x,
\end{array}
$$

where

$$
x_{2}=\left(\Delta T_{U}+\frac{1}{2} T_{g}\right) / B, \quad B=(2 k T m)^{1 / 2} / q E_{s},
$$

and

$$
x_{1}=\left(\Delta T_{U}-\frac{1}{2} T_{g}\right) / B .
$$

Values for the integrals in Eq. (22) appear in standard mathematical tables. By assuming a value for the initial energy (temperature), one fixes the parameter $B$. From the mathematical tables, then, one can construct

TABLE II. Translational energy of $\mathrm{CH}_{3}{ }^{+}$ fragment ion from $\mathrm{C}_{3} \mathrm{H}_{8}$.

\begin{tabular}{lccc}
\hline \hline This work & Mohler et al.* & Stantonb & Bracher et al..$^{\circ}$ \\
\hline $2.21 \mathrm{eV}$ & $2.2 \mathrm{eV}$ & $2.3 \mathrm{eV}$ & $2.2 \mathrm{eV}$
\end{tabular}

a F. L. Mohler, V. H. Dibeler, and R. M. Reese, J. Chem. Phys. 22 394 (1954).

b Reference 23.

- Reference 19. 
the function $I\left(\Delta T_{U}\right)$ for a given value of $B$. By repeating the process for several different values of the initial energy, the corresponding ion signals can be constructed. By comparing the experimentally obtained ion signal with those calculated for several values of the initial energy, one can deduce what the initial translational energy of the ion must have been to give the observed signal.

\section{RESULTS}

\section{A. Thermal Ions}

The expected peak widths at half-height $\left(W_{1 / 2}\right)$ for ions of thermal energy (assuming a source temperature of $300^{\circ} \mathrm{K}$ ) have been calculated from Eq. (14) and from Eq. (22). Figure 4 compares the results of these calculations with a number of experimentally determined peak widths for ions over a wide mass range.

These calculations show that the gate width causes significant broadening of the narrowest ion peaks (those for ions of small mass and low energy). However, the excellent agreement between the peak widths observed and those calculated by means of Eq. (22) shows that the broadening effect of the gate width can be accurately calculated from Eq. (22).

Moreover, this instrumental factor becomes increasingly insignificant for thermal ions whose peak widths at half-height are greater than $25 \mathrm{nsec}$, which is approximately the width expected for thermal ions with a $\mathrm{m} / \mathrm{e}$ ratio of 28 . For thermal ions of greater mass, the effect of the gate width may be neglected and the peak width may be accurately predicted from Eq. (14). The gate effect may likewise be neglected for ions formed with sufficient energy to have a peak of halfwidth greater than $25 \mathrm{nsec}$, for these the peak width may be calculated from Eq. (19).

\section{B. Excess-Energy Ions}

In addition to the ordinary quasithermal methyl ion peaks, many workers ${ }^{14,19-23}$ have also observed "satellite peaks" in the mass spectra of many organic compounds. These satellite peaks are produced by methyl ions formed with relatively large amounts in initial translational energy $(2-3 \mathrm{eV})$.

$19 \mathrm{~J}$. Bracher, H. Eberhardt, R. Fuchs, O. Osberghaus, and R. Taubert, Advan. Mass Spectry. 2, 285 (1962).

${ }^{20} \mathrm{P}$. Kusch, A. Hustrulid, and J. T. Tate, Phys. Rev. 52, 843 (1937).

${ }_{21}$ J. A. Hipple, R. E. Fox, and E. U. Conson, Phys. Rev. 69, 347 (1946)

${ }^{22}$ J. Olmsted, III, K. Street, Jr., and A. S. Newton, Phys. Rev. 40, 2114 (1964).

${ }^{23}$ H. E. Stanton, J. Chem. Phys. 30, 1116 (1959).
Figure 5 shows a chart recording of the methyl ion peak obtained from the dissociation of ethyl bromide. In addition to the relatively narrow peak caused by ions with a quasithermal energy distribution, there is a much wider peak produced by ions with an obviously quite different energy distribution. It should be noted that the high-energy methyl ions produce a symmetric peak approaching a rectangular shape, whose center appears at exactly the same mass number as the center of the peak produced by the quasithermal group of methyl ions. That is, the most probable flight time is $T\left(0, s_{0}\right)$ for both types of methyl ions.

The methyl ion from ethyl bromide has been investigated previously by other workers ${ }^{22}$ using a somewhat different method of determining initial translational energies. Table I compares their results with those we have obtained. It should be noted that our value for the high-energy process is for maximum translational energy and will be comparable to that of Olmsted et al., ${ }^{22}$ only if this process results in very energetic ions having a narrow distribution of energies. Figure 5 suggests that this may be the case.

The methyl ion from propane is also formed with two entirely different energy distributions. Table II compares the value we obtained for the initial translational energy of the high energy fragment with the values obtained by several other workers.

\section{DISCUSSION}

The good agreement between values obtained by this method and the values obtained by other workers confirms the usefulness of this method in accurately determining initial translational energy values up to several electron volts. Moreover, the simplicity of the calculations and the fact that the instrument need not be seriously modified permit the relatively quick and reliable determinations of excess translational energies. Consequently, this method should prove to be a useful tool in the investigation of such phenomena as the dissociation of diatomic molecules, the formation and dissociation of doubly charged polyatomic molecules, and the formation of negative ions.

\section{ACKNOWLEDGMENTS}

The authors wish to express their thanks for the support of the Robert A. Welch Foundation. Also, one of us (PMH) would like to acknowledge the support of the National Aeronautics and Space Administration in the form of a NASA Fellowship. 